\title{
Igniting homogeneous nucleation
}

\author{
J. C. Neu* \\ Department of Mathematics, University of California at Berkeley, Berkeley, California 94720, USA
}

L. L. Bonilla ${ }^{\dagger}$

Escuela Politécnica Superior, Universidad Carlos III de Madrid, Avenida de la Universidad 30, E-28911 Leganés, Spain

A. Carpio

Departamento de Matemática Aplicada, Universidad Complutense de Madrid, E-28040 Madrid, Spain

(Received 30 June 2004; published 8 February 2005)

\begin{abstract}
Transient homogeneous nucleation is studied in the limit of large critical sizes. Starting from pure monomers, three eras of transient nucleation are characterized in the classic Becker-Döring kinetic equations with two different models of discrete diffusivity: the classic Turnbull-Fisher formula and an expression describing thermally driven growth of the nucleus. The latter diffusivity yields time lags for nucleation which are much closer to values measured in experiments with disilicate glasses. After an initial stage in which the number of monomers decreases, many clusters of small size are produced and a continuous size distribution is created. During the second era, nucleii are increasing steadily in size in such a way that their distribution appears as a wave front advancing towards the critical size for steady nucleation. The nucleation rate at critical size is negligible during this era. After the wave front reaches critical size, it ignites the creation of supercritical clusters at a rate that increases monotonically until its steady value is reached. Analytical formulas for the transient nucleation rate and the time lag are obtained that improve classical ones and compare very well with direct numerical solutions.
\end{abstract}

DOI: 10.1103/PhysRevE.71.021601 PACS number(s): 82.70.Uv, 83.80.Qr, 05.40.-a, 05.20.Dd

\section{INTRODUCTION}

Homogeneous nucleation occurs in many examples of first order phase transitions [1] such as condensation of liquid droplets from a supersaturated vapor, glass-to-crystal transformations [2], crystal nucleation in undercooled liquids [3], and in polymers [4], colloidal crystallization [5], growth of spherical aggregates beyond the critical micelle concentration $(\mathrm{CMC})[6,7]$, and the segregation by coarsening of binary alloys quenched into the miscibility gap [8-10]. In condensed systems, a long time elapses before the nucleation rate (at which stable nucleii larger than the critical size are generated) reaches a steady state, therefore these systems offer excellent opportunities to study time-dependent nucleation [3].

Understanding the kinetics of nucleation and growth beyond the determination of the steady-state nucleation rate is a task of great importance and not yet completely accomplished. For example, it is desirable to obtain a simple asymptotic description of the transient until the steady-state nucleation stage sets in. Moreover, there is no clear distinction between nucleation and growth, and a unified theory of both processes does not exist [3] despite recent attempts at bridging the gap between nucleation and late-stage coarsening theories [11-13].

\footnotetext{
*Email address: neu@math.berkeley.edu

${ }^{\dagger}$ Author to whom all correspondence should be addressed. Email address: bonilla@ing.uc3m.es

${ }^{\ddagger}$ Email address: ana_carpio@mat.ucm.es
}

In this paper, we consider the problem of describing the approach to steady-state nucleation within the classical nucleation theory [3]. Thus our starting point is the BeckerDöring (BD) discrete kinetic model of nucleation and indefinite growth of a stable phase from a metastable state $[3,14,15]$. The BD model contains two kinetic rate constants that are related to each other by assuming detailed balance. To complete the description of the BD equations (BDE), a model for one of the rate constants, usually a discrete diffusivity describing the rate at which a cluster loses one monomer, is needed. In the classical theory, the discrete diffusivity is given by the Turnbull-Fisher (TF) expression which assumes that a monomer has to overcome an activation energy barrier for its transfer across the interface of a cluster. The TF discrete diffusivity is therefore proportional to the surface area of the cluster [16]. Other models are selected so as to yield the known expression for the adiabatic growth of a nucleus of critical size by either diffusion or by heat transfer. The discrete diffusivity of these later models is proportional to the cluster radius.

No matter which discrete diffusivity is used, starting from an initial condition of pure monomers surpassing the CMC, we expect that cluster size increases and stable supercritical nucleii are formed at a nucleation rate that will eventually become stationary at an exponentially small value. After the stationary nucleation has set in, the supercritical clusters continue growing, and the discrete diffusivity of the BDE can be ignored in the description of their growth, which is a pure convection in the space of cluster size. For precipitation processes, this will eventually result in late stage coarsening which we will not study in the present paper. 
The small parameter that informs our asymptotic analysis is $\epsilon=k_{c}^{-1 / 3}$, where $k_{c}$ is the number of monomers in the critical nucleus, a "large" quantity that ranges between 20 and 1000 for common materials [3]. Using other small parameters, such as the supersaturation, yields particular cases of our results (cf. Chap. 2 by Neu and Bonilla in Ref. [4]). The analysis of the BDE in the limit as $\epsilon \rightarrow 0$ distinguishes three well defined stages or eras in the approach to the stationary nucleation rate. Starting from the initial state of pure monomers, a continuous distribution of cluster sizes is established at the monomer's expense during the first era. During the second era, the clusters grow to the critical size in such a way that their size distribution is a traveling wave front in size space. As this wave reaches the critical size, the formation of supercritical nucleii starts, nucleation is ignited, and the nucleation rate increases from zero to its stationary value during the third era. We have obtained two different expressions for the nucleation rate (which is of paramount importance to compare with experiments): (I) a general expression in terms of the instantaneous location of the wave front and its instantaneous width, which solve two given differential equations, and (II) a more explicit description of the nucleation rate in terms of the solution of the linearized wave front position with an origin of time at the time $t_{M}$ needed for the exact wave front to advance from pure monomers to a certain near critical size. Numerical solution of the model confirms all the theoretical predictions.

Most previous studies of transient nucleation considered the Zeldovich-Frenkel equation (ZFE), which is a FokkerPlanck-type equation resulting from taking the continuum limit of the BDE [17]. Zeldovich [17] set the discrete diffusivity equal to its value at the critical cluster size and used a parabolic approximation for the variation of the free energy. The resulting expression for the transient nucleation rate was rather inaccurate [2]. Until the mid 1980s, work on the ZFE was based on similarly uncontrolled approximations [18]. Some of them gave expressions for the nucleation rate and time lag close to the values obtained by numerically solving the BDE for particular parameter values, but were far off for other parameter ranges [19]. Asymptotic theories for the ZFE were elaborated later [20-22]. There are two main differences between asymptotic results obtained for the discrete $\mathrm{BDE}$ and those obtained for the continuum ZFE: (i) the time lags for transient nucleation are different, as explained by $\mathrm{Wu}$ [19], and (ii) the width of the wave front and the time to ignition are different (wider for the ZFE). Nevertheless, other magnitudes such as relaxation times and the stationary nucleation rate are the same for asymptotic approximations of both, the BDE and the ZFE. Thus our simplified theory (II) yields expressions for the nucleation rate that are similar to those found by Shneidman [21] and by Shi et al. [22], although their time lags differ from ours, as one would expect from Wu's arguments [19]. For large critical sizes, our approximation (I) is better.

The rest of the paper is as follows. In Sec. II, we review the Becker-Döring model for nucleation and growth of spherical aggregates with the Turnbull-Fisher (TF) discrete diffusivity [16]. The binding energy of the aggregate with $k$ monomers ( $k$ cluster) relative to isolated monomers in solution is $(k-1)$ times the monomer-monomer bond energy plus a term proportional to the surface area of the aggregate. Beyond a critical density no equilibrium size distribution exists and the aggregates grow indefinitely. The main results of our asymptotic analysis are derived in Sec. III and compared with the numerical solution of the BDE with the TF discrete diffusion coefficient describing devitrification of lithium disilicate glass. Our results compare favorably with previous theories based on the $\mathrm{ZFE}$, the continuum approximation of the BDE. However, when compared with experimental data for glass disilicate, the theoretical time lag is about 30 times smaller. To improve the agreement with experiments, we propose in Sec. IV a different discrete diffusion coefficient selected so as to yield the known expression for the adiabatic growth of a nucleus of critical size by heat transfer. The asymptotic theory for the resulting BDE is similar to that explained in Sec. III, and the resulting time lag is much closer to experimental data. Section $\mathrm{V}$ compares our asymptotic results for the transient nucleation rate and for the number of supercritical clusters to previously known analytical formulas (unfortunately all of them dealing with the continuum ZFE, not with the discrete BDE as ours do) [20-24]. Technical matters are relegated to the Appendixes.

\section{KINETIC EQUATIONS AND STATIONARY SOLUTIONS}

The model presented here is nucleation in a lattice in which there are many more binding sites, $M$, than particles, $N$ [7]. We shall consider the thermodynamic limit, $N \rightarrow \infty$, with fixed particle density per site, $\rho \equiv N / M$. Let $p_{k}$ be the number of clusters with $k$ particles or, in short, $k$ clusters, and let $\rho_{k} \equiv p_{k} / M$ be the density of $k$ clusters. Note that the number densities per site, $\rho$ and $\rho_{k}$, are both dimensionless. Number densities per unit volume are obtained dividing $\rho$ and $\rho_{k}$ by the molecular volume, $v=V / M$. Particle conservation implies that the total particle density $\rho$ is constant:

$$
\sum_{k=1}^{\infty} k \rho_{k}=\rho .
$$

In the Becker-Döring kinetic theory of nucleation, a $k$ cluster can grow or decay by capturing or shedding one monomer at a time. Then [7]

$$
\begin{gathered}
\dot{\rho}_{k}=j_{k-1}-j_{k} \equiv-D_{-} j_{k}, \quad k \geqslant 2, \\
j_{k}=d_{k}\left\{e^{\left(D_{+} \varepsilon_{k}\right) / k_{B} T} \rho_{1} \rho_{k}-\rho_{k+1}\right\} .
\end{gathered}
$$

The monomer density $\rho_{1}$ can be obtained from the conservation identity (1) that relates it to the other cluster densities. In these equations, $\dot{\rho}_{k}=d \rho_{k} / d t$ and $D_{ \pm} u_{k} \equiv \pm\left[u_{k \pm 1}-u_{k}\right]$ are finite differences. $t, d_{k}$ and $j_{k}$ are nondimensional. $t$ and $d_{k}$ are related to the dimensional time $t^{*}$ and decay coefficient $d_{k}^{*}$ as follows:

$$
t=\Omega t^{*}, \quad d_{k}=\frac{d_{k}^{*}}{\Omega} .
$$

Here the factor $\Omega$ has units of frequency, it depends on the particular model we choose for $d_{k}$, and will be determined later. $j_{k}$ is the net rate of creation of a $k+1$ cluster from a $k$ 
cluster (the flux in size space), given by the mass action law. In Eq. (3) we have made the detailed balance assumption to relate the kinetic coefficient for monomer aggregation to that of decay of a $(k+1)$ cluster, $d_{k}$. This implies that the equilibrium size distribution solving $j_{k}=0$ has the form

$$
\tilde{\rho}_{k}=\rho_{1}^{k} \exp \left(\frac{\varepsilon_{k}}{k_{B} T}\right) .
$$

In Eqs. (3) and (5), $\varepsilon_{k}$ is the binding energy of a $k$ cluster, required to separate it into its monomer components. Then the total energy measured with respect to a configuration in which all clusters are monomers is $-\sum_{k=2}^{N} p_{k} \varepsilon_{k}$. For spherical aggregates,

$$
\varepsilon_{k}=\left((k-1) \alpha-\frac{3}{2} \sigma\left(k^{2 / 3}-1\right)\right) k_{B} T .
$$

This formula holds for $k \gg 1$, but we shall use it for all $k$ $\geqslant 1 . \alpha k_{B} T$ is the monomer-monomer bonding energy [6] which, in the case of precipitation of crystals from a solution or segregation by coarsening of binary alloys, may depend on the particle density $\rho$ (volume fraction) through some empirical formulas [14]. In Eq. (6), $\sigma$ $=2 \gamma_{s}\left(4 \pi v^{2} / 3\right)^{1 / 3} /\left(k_{B} T\right)$, where $\gamma_{s}$ and $v=V / M$ are the interfacial free energy per unit area (surface tension) and the molecular volume, respectively. Note that $\alpha$ and $\sigma$ are both dimensionless. The correction $3 \sigma k_{B} T / 2$ in Eq. (6) ensures that $\varepsilon_{1}=0$, and it improves the agreement between the nucleation rate obtained from the BDE and experiments [19]. More precise atomic models were proposed by Penrose $\mathrm{et} \mathrm{al}$. [14].

Equations (1)-(3) and (6) and a given discrete diffusivity $d_{k}$ form a closed system of equations that we can solve for an appropriate initial condition. If initially only monomers are present, we have $\rho_{1}(0)=\rho$, and $\rho_{k}(0)=0$ for $k \geqslant 2$. Before we obtain formulas for the kinetic coefficient $d_{k}$, we shall recall the more salient features of the equilibrium size distribution.

\section{A. Equilibrium size distribution}

The equilibrium distribution (5) satisfies $j_{k}=0$ and it can be written as

$$
\begin{gathered}
\widetilde{\rho}_{k}=\rho_{1} e^{-g_{k}}, \\
g_{k} \equiv-(k-1) \ln \rho_{1}-\frac{\varepsilon_{k}}{k_{B} T}=\frac{3}{2} \sigma\left(k^{2 / 3}-1\right)-(k-1) \ln \left(e^{\alpha} \rho_{1}\right),
\end{gathered}
$$

where $g_{k}$ is the activation energy, equivalently given by

$$
\begin{gathered}
g_{k}=\sigma_{k}-(k-1) \varphi, \quad \sigma_{k}=\frac{3}{2} \sigma\left(k^{2 / 3}-1\right) \quad(k \geqslant 1), \\
\varphi=\ln \left(e^{\alpha} \rho_{1}\right) .
\end{gathered}
$$

Here $\sigma_{1}=0=g_{1}$. Assuming $k \gg 1, g_{k}$ achieves its global maximum $g_{m}=\sigma k_{c}^{2 / 3} / 2+\sigma k_{c}^{-1 / 3}-3 \sigma / 2$ at the critical size
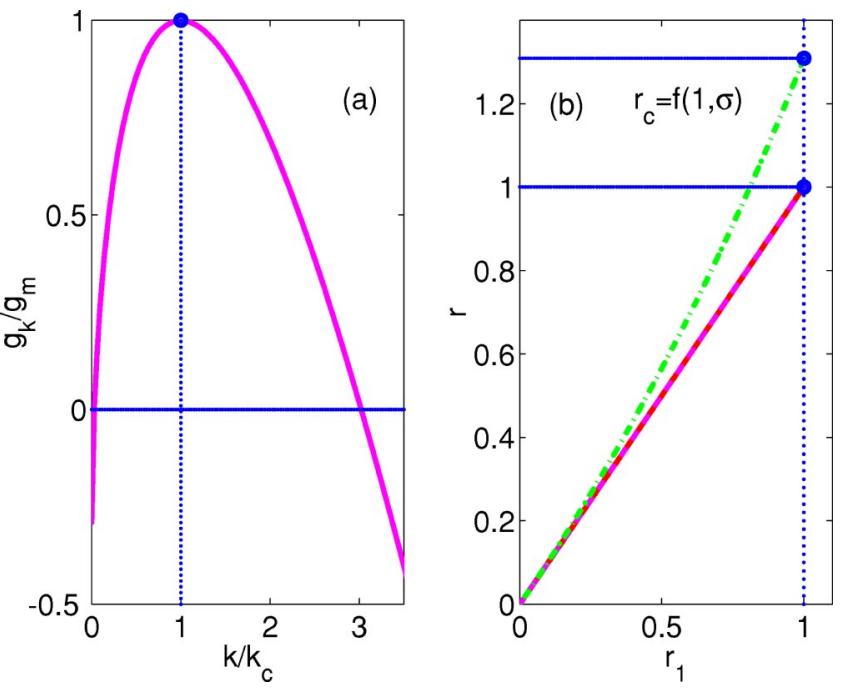

FIG. 1. (a) Scaled activation energy $g_{k} / g_{m}$ as a function of the scaled size $k / k_{c}$. (b) Scaled dimensionless density $r=\rho e^{\alpha}$ as a function of the scaled dimensionless monomer density $r_{1}=\rho_{1} e^{\alpha}$ for the equilibrium distribution (solid line). Data correspond to liquid iron at maximum undercooling (dot-dashed line), whereas for disilicate glass, $\rho \approx \rho_{1}$ (solid line).

$$
k=k_{c} \equiv\left(\frac{\sigma}{\varphi}\right)^{3}
$$

Equation (9) can be rewritten as

$$
g_{k} \sim \sigma k_{c}^{2 / 3}\left\{\frac{3}{2}\left(\frac{k}{k_{c}}\right)^{2 / 3}-\frac{k}{k_{c}}\right\}+\sigma k_{c}^{-1 / 3}-\frac{3 \sigma}{2} .
$$

$g_{k} / g_{m}$ as a function of $k / k_{c}$ is depicted in Fig. 1(a).

Rewriting the flux (3) in the BDEs in terms of the activation energy, we obtain

$$
j_{k}=d_{k}\left\{\left(e^{-D_{+} g_{k}}-1\right) \rho_{k}-D_{+} \rho_{k}\right\} .
$$

Equation (2) is a spatially discrete Smoluchowski equation with diffusion coefficient $d_{k}$ and drift velocity

$$
v_{k}=d_{k}\left(e^{-D_{+} g_{k}}-1\right) .
$$

Notice that $v_{k}<0$ for an activation energy that increases with $k$ and $v_{k}>0$ for decreasing $g_{k}$. Hence, $g_{k}$ indicates how the discrete advection $v_{k}$ transports the clusters in size space: subcritical clusters shrink as time elapses while supercritical clusters grow with time.

For the equilibrium densities (7), the conservation identity (1) becomes

$$
e^{\alpha} \rho=\sum_{k=1}^{\infty} k\left(e^{\alpha} \rho_{1}\right)^{k} e^{-\sigma_{k}}=\sum_{k=1}^{\infty} k e^{k \varphi-\sigma_{k}} .
$$

This series converges for $e^{\alpha} \rho_{1}=e^{\varphi} \leqslant 1(\varphi \leqslant 0)$, and diverges for $e^{\alpha} \rho_{1}>1(\varphi>0)$. At the critical micelle concentration (CMC), $\rho_{1}=e^{-\alpha}(\varphi=0)$, we obtain the critical density above which equilibrium is no longer possible, 
TABLE I. Data for lithium disilicate glass.

\begin{tabular}{|c|c|c|}
\hline Parameter & Symbol & Value \\
\hline Melting temperature & $T_{m}$ & $1300 \mathrm{~K}$ \\
\hline Entropy of fusion & $\Delta S_{f}$ & $40 \mathrm{~J} \mathrm{~mol}^{-1} \mathrm{~K}^{-1}$ \\
\hline Surface tension & $\gamma_{s}$ & $0.15 \mathrm{~J} / \mathrm{m}^{2}$ \\
\hline Preexponential diffusivity & $D_{0}$ & $2 \times 10^{9} \mathrm{~m}^{2} \mathrm{~s}^{-1}$ \\
\hline Activation energy for diffusion & $Q$ & $440 \mathrm{~kJ} / \mathrm{mol}$ \\
\hline Molecular volume & $v$ & $10^{-28} \mathrm{~m}^{3}$ \\
\hline TF time scale $(703 \mathrm{~K})$ & $\Omega_{T F}^{-1}$ & $0.613 \mathrm{~h}$ \\
\hline Heat capacity per unit volume & $\rho_{m} c$ & $10^{6} \mathrm{~J} \mathrm{~m}^{-3} \mathrm{~K}^{-1}$ \\
\hline Thermal conductivity $(703 \mathrm{~K})$ & $\rho_{m} c \kappa$ & $3.96 \times 10^{-18} \mathrm{~J} \mathrm{~m}^{-1} \mathrm{~s}^{-1} \mathrm{~K}^{-1}$ \\
\hline Thermally-driven-growth time scale (703 K) & $\Omega_{T D G}^{-1}$ & $6.196 \mathrm{~h}$ \\
\hline Critical size $(703 \mathrm{~K})$ & $k_{c}$ & 18 \\
\hline Undercooling (703 K) & $\widetilde{\varphi}$ & 4.087 \\
\hline Dimensionless surface tension $(703 \mathrm{~K})$ & $\sigma$ & 10.74 \\
\hline Dimensionless free energy barrier (703 K) & $g_{m}=\sigma / 2 k_{c}^{2 / 3}-3 \sigma / 2+\widetilde{\varphi}$ & 25.177 \\
\hline TF time scale $(820 \mathrm{~K})$ & $\Omega_{T F}^{-1}$ & $0.0478 \mathrm{~s}$ \\
\hline Thermal conductivity $(820 \mathrm{~K})$ & $\rho_{m} c \kappa$ & $1.84 \times 10^{-13} \mathrm{~J} \mathrm{~m}^{-1} \mathrm{~s}^{-1} \mathrm{~K}^{-1}$ \\
\hline Thermally-driven-growth time scale $(820 \mathrm{~K})$ & $\Omega_{T D G}^{-1}$ & $0.48 \mathrm{~s}$ \\
\hline Critical size $(820 \mathrm{~K})$ & $k_{c}$ & 34 \\
\hline Undercooling $(820 \mathrm{~K})$ & $\widetilde{\varphi}$ & 2.817 \\
\hline Dimensionless surface tension $(820 \mathrm{~K})$ & $\sigma$ & 9.207 \\
\hline Dimensionless free energy barrier $(820 \mathrm{~K})$ & $g_{m}=\sigma / 2 k_{c}^{2 / 3}-3 \sigma / 2+\tilde{\varphi}$ & 38.181 \\
\hline
\end{tabular}

$$
e^{\alpha} \rho_{c}=1+\sum_{k=2}^{\infty} k e^{-\sigma_{k}}
$$

For $\rho>\rho_{c}$, the BD kinetic equations predict phase segregation, i.e., indefinite growth of ever larger clusters.

\section{B. The controlling parameters}

The simplest nucleation problem consists of solving the BD equations (1), (2), and (13), with dimensionless activation energy $g_{k}=\sigma_{k}-(k-1) \varphi$, discrete diffusivity $d_{k}$ (to be chosen later) and initial conditions

$$
\rho_{1}(0)=\rho, \rho_{2}(0)=\rho_{3}(0)=\cdots=0 .
$$

The only parameters left in this initial value problem are $\rho$ and $\sigma . \rho$ controls the long-time behavior of the BDE: If $\rho$ $\leqslant \rho_{c}$ given by (16), $\rho_{k}(t)$ approach their equilibrium values (7), with monomer density $\rho_{1}$ that solves Eq. (1):

$$
\rho e^{\alpha}=f\left(\rho_{1} e^{\alpha} ; \sigma\right) \equiv \sum_{k=1}^{\infty} k\left(\rho_{1} e^{\alpha}\right)^{k} e^{-\sigma_{k}}
$$

The graph of this function is either the solid line or the dashed line in Fig. 1(b). If $\rho>\rho_{c}$, cluster sizes grow indefinitely whereas their density becomes small. Thus there remains a residual monomer concentration whose density $\rho_{1} e^{\alpha} \rightarrow 1$ as $t \rightarrow \infty$. Summarizing, the union of solid or dashed lines in Fig. 1(b) and the vertical line $\rho_{1} e^{\alpha}=1$ for $\rho>\rho_{c}$ represents the long-time limit of the monomer concentration as a function of $\rho$.
Let us identify the controlling parameters $\rho$ and $\sigma$ in a physical system undergoing homogeneous nucleation. A good experimental example is the transformation of certain silicate glasses to crystals (devitrification) [3]. In particular, abundant data exist for lithium disilicate and we have compiled in Table I appropriate values of parameters characterizing nucleation [2]. In disilicate, the free energy per molecule of the crystal phase in the activation energy (9) is proportional to the undercooling

$$
\varphi=\frac{\Delta S_{f}\left(T_{m}-T\right)}{N_{A} k_{B} T}
$$

where $T_{m}$ is the melting temperature, $\Delta S_{f}$ is the molar entropy of fusion, and $N_{A}$ is Avogadro's number. The dimensionless density $\rho=e^{\varphi(0)}$ can be extracted from Eq. (19) as explained in Sec. III. In energy units, the activation free energy is $k_{B} T g_{k}=\gamma_{s} 4 \pi a^{2}-k_{B} T \varphi k$, where $a$ is the radius of a spherical $k$ cluster. From the expression for the volume of this cluster, $k v=4 \pi a^{3} / 3$ ( $v$ is the molecular volume), we obtain $a=[3 v /(4 \pi)]^{1 / 3} k^{1 / 3}$, and therefore

$k_{B} T\left(g_{k}-\varphi+\frac{3 \sigma}{2}\right)=\gamma_{s}(4 \pi)^{1 / 3}(3 v)^{2 / 3} k^{2 / 3}-\Delta S_{f}\left(T_{m}-T\right) k / N_{A}$.

Comparing Eq. (20) with Eq. (9) yields $\sigma$ $=\left(32 \pi v^{2} / 3\right)^{1 / 3} \gamma_{s} /\left(k_{B} T\right)$, and the critical size 


$$
k_{c}^{1 / 3}=\left(\frac{32 \pi v^{2}}{3}\right)^{1 / 3} \frac{\gamma_{s} N_{A}}{\Delta S_{f}\left(T_{m}-T\right)} .
$$

The other parameters in Table I will be used later to model the discrete diffusivity in the BDE. We observe that the critical size increases with temperature: $k_{c}=18$ at $703 \mathrm{~K}$ and $k_{c}$ $=34$ at $820 \mathrm{~K}$. For other materials, such as undercooled liquid metals, critical sizes can be rather large: liquid iron at maximum undercooling has $k_{c}=494$, whereas $k_{c}=2253$ for liquid rutenium at maximum undercooling [3].

\section{Equivalent Becker-Döring system}

As they stand, the BDE are rather stiff and hard to solve numerically. For example, at equilibrium, Table I indicates that $\rho_{k_{c}} / \rho_{1}=e^{-g_{m}-\tilde{\varphi}} \approx e^{-25.2-4.1} \approx 2 \times 10^{-13}$ for disilicate glass at $703 \mathrm{~K}$, and $\rho_{k_{c}} / \rho_{1} \approx 1.6 \times 10^{-18}$ for disilicate glass at $820 \mathrm{~K}$. This motivates the following change of variable

$$
\rho_{k}=\rho_{1} e^{-g_{k}} s_{k}=e^{-\alpha} e^{k \varphi-\sigma_{k}} s_{k},
$$

according to Eq. (10). Note that $s_{k}=1$ in equilibrium. Since $g_{1}=0$, this equation implies

$$
s_{1} \equiv 1,
$$

for all $t$. For the initial condition (17), $e^{\varphi(0)-\alpha}=\rho_{1}(0)=\rho$, and the conservation identity (1) becomes

$$
e^{\varphi(0)}=e^{\varphi}+\sum_{k=2}^{\infty} k e^{k \varphi-\sigma_{k}} s_{k},
$$

in which we have used Eq. (22). In terms of the $s_{k}$, the flux can be written as

$$
j_{k}=d_{k} \exp \left[(k+1) \varphi-\sigma_{k+1}\right]\left(s_{k}-s_{k+1}\right),
$$

and the BDE (2) and (13) become

$$
\dot{s}_{k}+u_{k}\left(s_{k+1}-s_{k}\right)=-k \dot{\varphi} s_{k}+d_{k-1}\left(s_{k-1}-2 s_{k}+s_{k+1}\right),
$$

for $k \geqslant 2$. Here,

$$
u_{k}=d_{k-1}-d_{k} e^{\varphi-D_{+} \sigma_{k}} .
$$

The term $u_{k} D_{+} s_{k}$ in Eq. (26) represents discrete advection, with a drift velocity $u_{k}=-v_{k}+\left(d_{k-1}-d_{k}\right) \sim-v_{k}$, which is essentially minus the drift velocity in the original $\mathrm{BDE}$ for $k$ $\gg 1$. Thus the advection in Eq. (26) climbs up the activation energy barrier, from small values of $g_{k}$ to large ones.

In summary, the transformed nucleation initial-boundary value problem consists of the balance equations (26), the particle conservation equation (24), the boundary condition (23), $s_{1}=1$, and initial conditions $s_{k}(0)=0$ for all $k \geqslant 2$. Its solution gives $\varphi(t)$ and $s_{k}(t)$ for all $k \geqslant 2$ and all $t>0$.

\section{Stationary solution}

The stationary solution of the BDE has a flux independent of cluster size, so that $j_{k}=d_{k} \exp \left[(k+1) \varphi-\sigma_{k+1}\right]\left(s_{k}-s_{k+1}\right)=j$, from which $\left(s_{k+1}-s_{k}\right)=-j \exp \left[\sigma_{k+1}-(k+1) \varphi\right] / d_{k}$, and therefore

$$
s_{k}=1-j \sum_{l=1}^{k-1} \frac{\exp \left[\sigma_{l+1}-(l+1) \varphi\right]}{d_{l}},
$$

for $k \geqslant 2$. Since $s_{\infty}=0, j$ can be obtained from this expression in terms of an infinite series

$$
j=\frac{1}{\sum_{l=1}^{\infty} \exp \left[\sigma_{l+1}-(l+1) \varphi-\ln d_{l}\right]} .
$$

Substituting this expression back into Eq. (28), we obtain

$$
s_{k}=1-\frac{\sum_{l=1}^{k-1} \exp \left[\sigma_{l+1}-(l+1) \varphi-\ln d_{l}\right]}{\sum_{l=1}^{\infty} \exp \left[\sigma_{l+1}-(l+1) \varphi-\ln d_{l}\right]} .
$$

Then, $\rho_{k}=\rho_{1} e^{-g_{k}} s_{k}$.

\section{E. Turnbull-Fisher discrete diffusivity}

To solve the BDE, we need to establish reasonable models of the kinetic coefficient $d_{k}$ (discrete diffusivity) for the decay of the $(k+1)$ cluster. A classical formula due to Turnbull and Fisher [16] applies to spherical clusters whose growth is limited by the reaction rate at their boundary: $d_{k}^{*}$ is the product of the number of active sites on the aggregate times the molecular jump rate $[2,16]$

$$
d_{k}^{*}=4 k^{2 / 3} e^{D_{+} g_{k} / 2} \frac{6 D}{\lambda^{2}}=\Omega k^{2 / 3} e^{D_{+} g_{k} / 2}, \Omega^{-1}=\frac{v^{2 / 3}}{24 D} \equiv \frac{v^{2 / 3} e^{Q /(R T)}}{24 D_{0}} .
$$

Here $D=D_{0} e^{-Q /(R T)}$ is the diffusion coefficient in the liquid, $Q$ is the activation energy for diffusion (see Table $\mathrm{I}$ ), $R$ $=k_{B} N_{A}$ is the gas constant, and $\lambda=v^{1 / 3}$ ( $v$ is the molecular volume). If we nondimensionalize time as in Eq. (4) with this definition of $\Omega$, we obtain

$$
d_{k}=k^{2 / 3} e^{D_{+} g_{k} / 2} .
$$

\section{ASYMPTOTIC THEORY OF TRANSIENT HOMOGENEOUS NUCLEATION WITH THE TURNBULL- FISHER DIFFUSIVITY}

In this section, we shall interpret the numerical solutions shown in Figs. 2-4 by using singular perturbation methods. Our theory will be described using the TF discrete diffusivity (31) and compared to numerical solution of the BDE for the crystallization of disilicate glass at different undercoolings.

\section{A. Initial transient}

Initially, $\rho_{1}(0)=\rho$ and there are no multiparticle aggregates. There is an initial transient stage during which dimers, trimers, etc. form at the expense of the monomers. This initial stage is characterized by the decay of the chemical driving force $\varphi=\alpha+\ln \rho_{1}$ to a quasi-stationary value $\widetilde{\varphi}$, given by Eq. (19) in the case of disilicate glass, and the emergence of a continuum size distribution. Knowing this, we choose the initial chemical driving force $\varphi(0)$ so that the quasistation- 

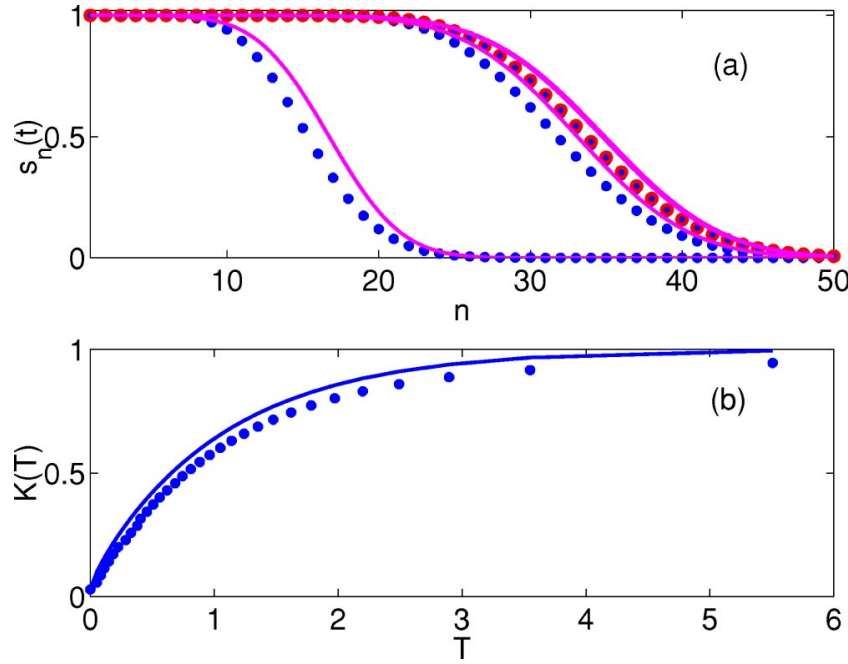

FIG. 2. (a) Comparison of $s_{n}(t)$ evaluated (at different times) from the numerical solution of the discrete equations (26) to the asymptotic result (54) (solid line). (b) $K(T)$ calculated from Eq. (41) with $K(0)=\epsilon^{3}$ (solid line) is compared to the numerically obtained position of the wave front. Data correspond to disilicate glass at $820 \mathrm{~K}$. All variables are written in dimensionless units.

ary value $\widetilde{\varphi}$ given by Eq. (19) is attained at the end of the initial stage.

In materials such as disilicate glass at the temperatures we consider, the critical size is relatively small. Then $\varphi(0) \approx \widetilde{\varphi}$, and the initial stage is very short. As the critical size increases (as in the case of undercooled liquid metals), $\varphi(0)$ may differ appreciably from $\widetilde{\varphi}$, and the initial stage lasts longer. However, even in such cases, the duration of the initial stage, $t_{\infty}$, is negligible if we are interested in the overall
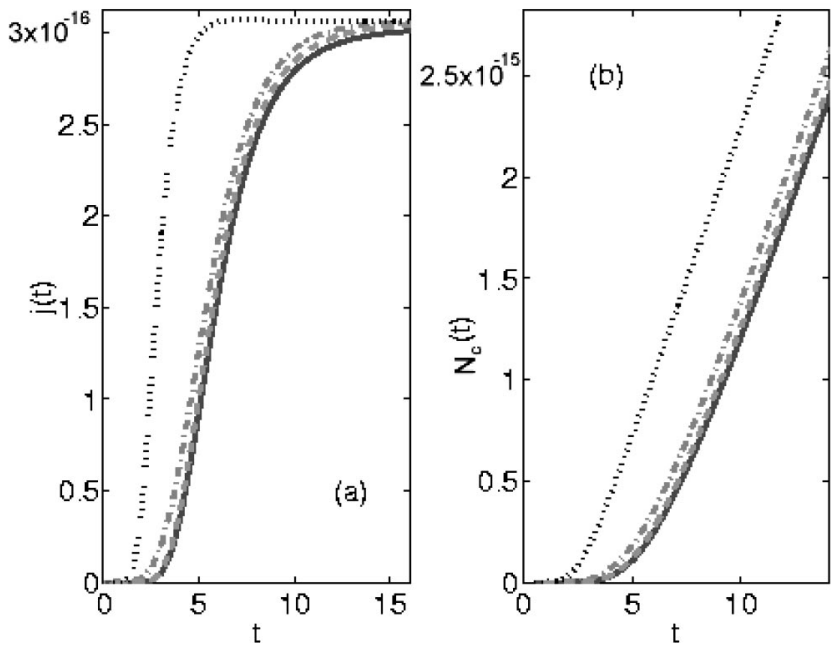

FIG. 3. (a) Evolution of the dimensionless flux at critical size $j(t)$, and (b) number of clusters surpassing critical size $N_{c}(t)$ as a function of dimensionless time for disilicate glass at $820 \mathrm{~K}, k_{c}$ $=34$. Solid lines correspond to numerical results, dashed lines to the approximation given by Eq. (58), dot-dashed lines to the linearization approximation (67), and dotted lines to the approximation (C8) corresponding to linearizing the equations for $K(T)$ and $A(T)$ as in Appendix C.
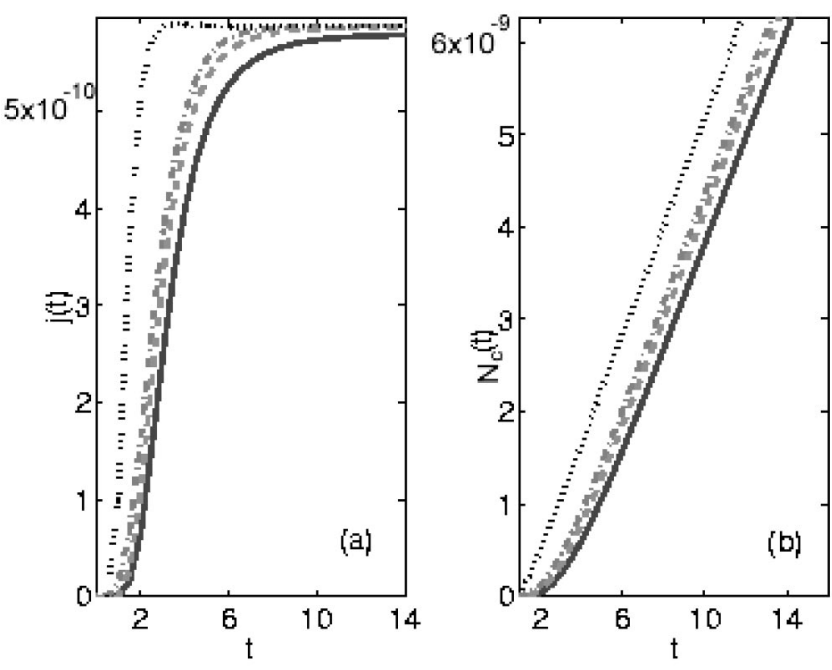

FIG. 4. Same as in Fig. 3 for disilicate glass at $703 \mathrm{~K}$, $k_{c}=18$.

duration of the transient stage to quasi-stationary nucleation. We shall show later that the duration of the initial stage compared to the duration of the overall transient is of order $k_{c}^{-2 / 3}$, a very small quantity for materials with large critical sizes.

\section{B. Wave front advancing towards the cluster of critical size}

After the first era, clusters of increasing size are formed. For sufficiently small clusters, the continuum size distribution approaches the equilibrium distribution with $\varphi=\tilde{\varphi}$. This situation can be observed as an advancing wave front in the variable $s_{k}(t)$, satisfying $s_{k} \sim 1$ (equilibrium) behind the front and $s_{k} \sim 0$ ahead of the front. This second era is described by Eqs. (24)-(27) with $\varphi=\widetilde{\varphi}$ and $\dot{\varphi}=0$. The critical sizes,

$$
k_{c}=\left(\frac{\sigma}{\tilde{\varphi}}\right)^{3},
$$

for disilicate glass are relatively small, between 10 and 50, but they are large for undercooled liquid metals, generally between 100 and 1000 . Hence we shall use as a small gauge parameter

$$
\epsilon=\frac{\widetilde{\varphi}}{\sigma}
$$

Our asymptotic analysis will be carried out in the limit $\epsilon$ $\rightarrow 0$, and therefore $k_{c}=\epsilon^{-3} \rightarrow \infty$. Then $d_{k}, u_{k}$, and $\sigma_{k}$ in Eqs. (32), (26), and (27) are smooth functions of $k>0$ :

$$
\begin{gathered}
d(k)=k^{2 / 3} e^{\left[D_{+} \sigma(k)-\tilde{\varphi}\right] / 2}, \quad \sigma(k)=\frac{3}{2} \sigma\left(k^{2 / 3}-1\right), \\
u(k)=d(k-1)-d(k) \exp [\tilde{\varphi}-\sigma(k+1)+\sigma(k)] .
\end{gathered}
$$

\section{Position of the wave front}

In the numerical solutions shown in Fig. 2(a), the graphs of $s_{k}$ vs $k$ at fixed time have clear inflection points at some $k$, 
where $s_{k} \approx 1 / 2$. The inflection point is taken as the position of the wave front. In the continuum model, the front position $k=k_{f}(t)$ is a smooth function which obeys

$$
\dot{k}_{f}=u\left(k_{f}\right) .
$$

Scaling $k_{f}$ as

$$
k_{f}=\frac{K}{\epsilon^{3}}
$$

(same scaling as $k_{c}=\epsilon^{-3}$ ), the right-hand side of Eq. (37) becomes

$$
\begin{gathered}
u\left(k_{f}\right)=\frac{1}{\epsilon^{2}} U(K)+O(\epsilon), \\
U(K)=2 K^{2 / 3} \sinh \left(\frac{\widetilde{\varphi}}{2}\left(K^{-1 / 3}-1\right)\right) .
\end{gathered}
$$

Equation (37) can be rewritten as

$$
\frac{d K}{d T}=U(K) \equiv 2 K^{2 / 3} \sinh \left(\frac{\widetilde{\varphi}}{2}\left(K^{-1 / 3}-1\right)\right),
$$

provided we define the slowly varying time scale $T=\epsilon t$, and take the limit as $\epsilon \rightarrow 0$. Figure 2(b) compares the position of the wave front calculated by solving Eq. (41) with $K(0)=\epsilon^{3}$ to the value obtained from the numerical solution of Eq. (26). Note that the solution of Eq. (41) presents a time shift with respect to the numerical solution of the discrete model. This time shift reflects the breakdown of the continuum limit as $K \rightarrow 0$, due to discreteness, and also the transient in $\varphi(t)$ before it settles to $\widetilde{\varphi}$. If the solution of Eq. (41) is forced to agree with the numerical $K(T)$ when the latter is, say, 0.1, the comparison fares much better.

\section{Shape of the wave front}

The leading edge of the wave front is a layer centered at $K(T)$ in which $s_{k}$ decreases from 1 to 0 as $k$ increases through it. The continuum representation of $s_{k}$ in this layer is

$$
s_{k}=S(X, T ; \epsilon),
$$

where $S$ is a smooth function of its arguments and $X$ is the scaled displacement from the wave front location at $k$ $=K / \epsilon^{3}$, i.e.,

$$
X=\epsilon^{p}\left(k-\frac{K}{\epsilon^{3}}\right) .
$$

The scaling exponent $p$, presumably with $0<p<3$, is to be determined. The description (42) and (43) should hold as $\epsilon$ $\rightarrow 0$ with $X$ fixed, so that the layer thickness scales as $\epsilon^{-p}$. Substituting Eq. (42) into Eq. (26) yields

$$
\begin{aligned}
\epsilon \frac{\partial S}{\partial T}-\epsilon^{p-2} \frac{d K}{d T} \frac{\partial S}{\partial X}+u\left(\frac{K}{\epsilon^{3}}+\frac{X}{\epsilon^{p}}\right)\left[S\left(X+\epsilon^{p}, T ; \epsilon\right)-S(X, T ; \epsilon)\right] \\
=d\left(\frac{K}{\epsilon^{3}}+\frac{X}{\epsilon^{p}}\right)\left[S\left(X-\epsilon^{p}, T ; \epsilon\right)-2 S(X, T ; \epsilon)+S(X\right. \\
\left.\left.+\epsilon^{p}, T ; \epsilon\right)\right] .
\end{aligned}
$$

Carrying out the straightforward expansion in powers of $\epsilon$, Eq. (44) adopts the following asymptotic form

$$
\begin{aligned}
\epsilon & \frac{\partial S}{\partial T}+\epsilon^{p-2}\left[U(K)-\frac{d K}{d T}\right] \frac{\partial S}{\partial X}+\epsilon U^{\prime}(K) X \frac{\partial S}{\partial X} \\
& =\epsilon^{2 p-2}\left[K^{2 / 3} e^{\widetilde{\varphi}\left(K^{-1 / 3}-1\right) / 2}-\frac{1}{2} U(K)\right] \frac{\partial^{2} S}{\partial X^{2}}+o\left(\epsilon^{2 p-2}\right),
\end{aligned}
$$

as $\epsilon \rightarrow 0$ with $X, K$ fixed. Here $U(K)$ is given by Eq. (40). To obtain Eq. (45), we have used Eqs. (38) and (39):

$$
\begin{aligned}
u\left(\frac{K+\epsilon^{3-p} X}{\epsilon^{3}}\right) & =\epsilon^{-2} U\left(K+\epsilon^{3-p} X\right)+O(\epsilon) \\
& =\epsilon^{-2} U(K)+\epsilon^{1-p} U^{\prime}(K) X+o\left(\epsilon^{1-p}\right) .
\end{aligned}
$$

The dominant balance of diffusion and convection in Eq. (45) yields $2 p-2=1$, or $p=3 / 2$. Hence Eq. (43) yields

$$
X=\epsilon^{3 / 2}\left(k-\frac{K}{\epsilon^{3}}\right),
$$

and the limit of Eq. (45) as $\epsilon \rightarrow 0$ is

$$
\begin{gathered}
\frac{\partial S}{\partial T}+U^{\prime}(K) X \frac{\partial S}{\partial X}=D(K) \frac{\partial^{2} S}{\partial X^{2}}, \\
D(K) \equiv \lim _{\epsilon \rightarrow 0}\left[d\left(\epsilon^{-3} K\right)-\frac{1}{2} u\left(\epsilon^{-3} K\right)\right] \epsilon^{2} \\
=K^{2 / 3} \cosh \left(\frac{\widetilde{\varphi}}{2}\left(K^{-1 / 3}-1\right)\right) .
\end{gathered}
$$

Had we carried out the same analysis for the ZFE, we would have found $D(K) \sim d\left(\epsilon^{-3} K\right) \epsilon$. This would have resulted in a wider wave front and a longer time to ignition than those described below.

\section{Flux and wave front width}

Besides determining the shape of the wave front near its location, Eq. (47) yields the behavior of the flux (creation rate of clusters larger than $k$ ) $j_{k}$ near $k=k_{f}$. If we substitute Eqs. (32), (42), and (46) into Eq. (25):

$$
\begin{aligned}
j_{k}= & -d_{k} e^{(k+1) \tilde{\varphi}-\sigma_{k+1} D_{+} s_{k}} \\
= & -k^{2 / 3} \exp \left[\left(k+\frac{1}{2}\right) \tilde{\varphi}\right. \\
& \left.-\frac{3 \sigma}{4}\left[(k+1)^{2 / 3}+k^{2 / 3}\right]+\frac{3 \sigma}{2}\right] D_{+} s_{k},
\end{aligned}
$$

we obtain

$$
\begin{aligned}
j_{k} \sim \epsilon^{-1 / 2} K^{2 / 3} e^{3 \widetilde{\varphi} /(2 \epsilon)} & \\
& \times \exp \left[-\frac{G(K)}{\epsilon^{3}}-\frac{G^{\prime}(K) X}{\epsilon^{3 / 2}}-\frac{G^{\prime}(K)}{2}-\frac{G^{\prime \prime}(K)}{2} X^{2}\right] \frac{\partial S}{\partial X} .
\end{aligned}
$$

Here 


$$
G(K) \equiv \widetilde{\varphi}\left(\frac{3}{2} K^{2 / 3}-K\right)
$$

is a scaled version of the activation energy (9).

Since $j_{k}$ is proportional to $\partial S / \partial X$, it is convenient to differentiate Eq. (47) with respect to $X$ in order to obtain an equation for $J \equiv-\partial S / \partial X$,

$$
\frac{\partial J}{\partial T}+U^{\prime}(K) \frac{\partial(X J)}{\partial X}=D(K) \frac{\partial^{2} J}{\partial X^{2}} .
$$

Notice that $J$ is locally conserved, and the following integral conservation identity holds:

$$
1=-[S]_{-\infty}^{\infty}=-\int_{-\infty}^{\infty} \frac{\partial S}{\partial X} d X=\int_{-\infty}^{\infty} J d X .
$$

Equation (51) has Gaussian solutions satisfying Eq. (52),

$$
J(X, T)=\frac{1}{2 \sqrt{\pi A(T)}} \exp \left[-\frac{X^{2}}{4 A(T)}\right],
$$

which yields

$$
S(X, T)=\frac{1}{2} \operatorname{erfc}\left[\frac{X}{2 \sqrt{A(T)}}\right]
$$

for the wave front profile. Inserting Eq. (53) in Eq. (51), we find the following equation for $A(T)>0$ :

$$
\frac{d A}{d T}-2 U^{\prime}(K) A=D(K) .
$$

Since $K(T)$ is an increasing function, we can express $A$ as a function of $K$. Inserting Eq. (41) in Eq. (55), we get

$$
\frac{d A}{d K}-\frac{2 U^{\prime}(K)}{U(K)} A=\frac{D(K)}{U(K)} .
$$

Direct integration of this equation yields

$$
A=q U^{2}+U^{2} \int D \frac{d K}{U^{3}}=\frac{3 K^{4 / 3}\left(1+q \sinh ^{2}\left[\frac{\widetilde{\varphi}\left(K^{-1 / 3}-1\right)}{2}\right]\right)}{2 \widetilde{\varphi}},
$$

in which $q$ is an arbitrary constant.

After insertion of Eq. (53), the flux (49) becomes

$$
\begin{aligned}
j_{k} \sim & \frac{K^{2 / 3} e^{3 \tilde{\varphi} /(2 \epsilon)}}{2 \sqrt{\epsilon \pi A}} \exp \left\{-\frac{G(K)}{\epsilon^{3}}-\frac{G^{\prime}(K) X}{\epsilon^{3 / 2}}-\frac{G^{\prime}(K)}{2}\right. \\
& \left.-\left[\frac{G^{\prime \prime}(K)}{2}+\frac{1}{4 A}\right] X^{2}\right\} .
\end{aligned}
$$

Here $K=K(T)$ and $A=A(T)$ are found by solving the differential equations (41) and (55) with initial conditions $K(0)$ $=\epsilon^{3}$ and $A(0)=3 \epsilon^{4} /(2 \widetilde{\varphi})$, respectively. We have to set $q=0$ in Eq. (57) for $A$ would become exponentially large as $K=\epsilon^{3}$ $\rightarrow 0$ otherwise. As $T \rightarrow \infty, K \rightarrow 1$ and $A \rightarrow-D(1) /\left[2 U^{\prime}(1)\right]$. The definitions (40) and (48) of $U(K)$ and $D(K)$ imply $U^{\prime}(1)=-\tilde{\varphi} / 3, D(1)=1$. Hence, $A \rightarrow 3 /(2 \widetilde{\varphi})$ as $T \rightarrow \infty$, or as $K \rightarrow 1$ in Eq. (57). The definition (50) of $G(K)$ implies
$G(1)=\tilde{\varphi} / 2, G^{\prime}(1)=0$, and $G^{\prime \prime}(1)=-\widetilde{\varphi} / 3$. Hence, the limit as $T \rightarrow \infty$ of the creation rate (58) is

$$
j_{k} \sim j_{\infty} \equiv \sqrt{\frac{\tilde{\varphi}}{6 \pi \epsilon}} \exp \left(-\frac{\tilde{\varphi}}{2 \epsilon^{3}}+\frac{3 \tilde{\varphi}}{2 \epsilon}\right) .
$$

Notice that the terms proportional to $X$ and $X^{2}$ have disappeared from this expression and therefore $j_{\infty}$ is asymptotically uniform for $X=O(1)$. Equation (59) is the classical quasisteady nucleation rate of supercritical clusters due to Zeldovich [17], and it can be directly obtained from the stationary flux (29) in the limit as $\epsilon \rightarrow 0$.

\section{The nucleation rate of supercritical clusters}

Let us now study the transient creation rate, in which $j$ $\equiv j_{k_{c}}$ increases from 0 to the steady Zeldovich value (59). As we have just seen, our theory predicts that the wave front profile is given by Eq. (54), where $K(T)$ and $A(T)$ are solutions of Eqs. (41) and (55), respectively. The flux of clusters with sizes larger than $k$ is then given by Eq. (58). Setting $k$ $=k_{c}=\epsilon^{-3}$ (critical size) and $X=(1-K(T)) / \epsilon^{3 / 2}$ in this equation, we obtain the nucleation rate predicted by our theory, $j(t)$. Its integral over time yields the number of supercritical clusters, $N_{c}(t)$. We shall consider now a different and more explicit approximation of these results.

\section{Linearization of the wave front speed about the critical size}

Let us fix $k=k_{c}=\epsilon^{-3}$ (critical size) in the definition (46) of $X$ :

$$
X=\frac{1-K}{\epsilon^{3 / 2}} \equiv \kappa .
$$

We now set $X=\kappa$ in Eq. (58) and perform the limit as $\epsilon$ $\rightarrow 0$ with $\kappa$ fixed. The result is

$$
j \sim j_{\infty} e^{-\widetilde{\varphi} \kappa^{2} / 6-\epsilon^{3 / 2} \widetilde{\varphi} \kappa / 6} \sim j_{\infty} e^{-\widetilde{\varphi} \kappa^{2} / 6},
$$

provided we use the limiting stationary value $(4 A)^{-1}$ $=-G^{\prime \prime}(1) / 2$.

The transient turns on when $\kappa \equiv(1-K) / \epsilon^{3 / 2}=O(1)$. Since $U\left(1-\epsilon^{3 / 2} \kappa\right) \sim \epsilon^{3 / 2} \widetilde{\varphi} \kappa / 3$, the wave front equation (40) yields

$$
\frac{d \kappa}{d T}=-\frac{\widetilde{\varphi}}{3} \kappa,
$$

as $\epsilon \rightarrow 0$. The solution of this equation is

$$
\begin{gathered}
\kappa=\kappa_{M} e^{-\widetilde{\varphi} e^{\widetilde{\varphi}}\left(T-T_{M}\right) / 3}=\kappa_{M} e^{-\left(t-t_{M}\right) /(2 \tau)}, \\
\tau^{-1}=\frac{2}{3} \widetilde{\varphi} \epsilon .
\end{gathered}
$$

It is convenient to choose $\kappa_{M}$ as the value of $\kappa$ at which the flux $j$ reaches its inflection point. Then we may consider that the wave front has ignited the nucleation of supercritical clusters. Straightforward use of Eqs. (61) and (62) shows that

$$
\kappa_{M}=\sqrt{\frac{6}{\tilde{\varphi}}} .
$$


Moreover, $T_{M}=\epsilon t_{M}$ is the time to ignition, at which the wave front $K(T)$ reaches the value $K=1-\epsilon^{3 / 2} \kappa_{M}$. From Eq. (41), we obtain

$$
\begin{aligned}
t_{M}= & t_{\infty}+\frac{3}{2 \widetilde{\varphi} \epsilon}\left\{\ln \left(\frac{\widetilde{\varphi}\left(1-\epsilon^{3}\right)^{2}}{6 \epsilon^{3}}\right)\right. \\
& \left.+\int_{\epsilon^{3}}^{1-\epsilon^{3 / 2} \kappa_{M}}\left[\frac{\tilde{\varphi}}{3 K^{2 / 3} \sinh \left[\frac{\tilde{\varphi}}{2}\left(K^{-1 / 3}-1\right)\right]}+\frac{2}{K-1}\right] d K\right\},
\end{aligned}
$$

where $t_{\infty}$ is the duration of the initial stage. We could have expanded the integral in this expression, but Eq. (66) is better suited for numerical calculation. The nucleation rate is found by inserting Eq. (63) in Eq. (61):

$$
j \sim j_{\infty} \exp \left[-e^{-\left(t-t_{M}\right) / \tau}\right],
$$

in which a term of order $\epsilon^{3 / 2}$ has been ignored in the exponential.

Integrating $j(t)$ over time, we find the number of supercritical clusters as a function of time. In the limit as $t \rightarrow \infty$, this number is $N_{c}(t) \sim j_{\infty}(t-\theta)$, where the time lag $\theta$ is approximately given by $\theta=t_{M}+\tau \gamma+\tau E_{1}\left(e^{t_{M} / \tau}\right)$, or

$$
\begin{aligned}
\theta= & t_{\infty}+\frac{3}{2 \widetilde{\varphi} \epsilon}\left\{\ln \left(\frac{\tilde{\varphi}\left(1-\epsilon^{3}\right)^{2}}{6 \epsilon^{3}}\right)+\gamma+\tau E_{1}\left(e^{t_{M} / \tau}\right)\right. \\
& \left.+\int_{\epsilon^{3}}^{1-\epsilon^{3 / 2} \kappa_{M}}\left[\frac{\tilde{\varphi}}{3 K^{2 / 3} \sinh \left[\frac{\tilde{\varphi}}{2}\left(K^{-1 / 3}-1\right)\right]}+\frac{2}{K-1}\right] d K\right\},
\end{aligned}
$$

where $\gamma=0.577215 \ldots$ is Euler's constant and $E_{1}(x)$ is an exponential integral, see the derivation in Appendix B. The time lag $\theta$ can be directly compared to experimental values [3].

\section{Comparison between different approximations}

Figure 3(a) compares $j(t)$ calculated from the numerical solution of the BDE for devitrification of disilicate glass at $820 \mathrm{~K}$, from Eqs. (67) and (66) with $t_{\infty}=0$, and from Eq. (58) with $X=(1-K(T)) / \epsilon^{3 / 2}$. We find that the more precise expression, Eq. (58), captures better the width and location of the transition region between $j=0$ and $j=j_{\infty}$, as compared with the simple approximation given by Eqs. (67) and (66). Both approximations present a small overshoot and yield a smaller time lag $\theta$ than that obtained from the numerical solution of the BDE. The overshoot decreases as the critical size increases. Another approximation consists of linearizing the equations for $K(T)$ and $A(T)$ about the critical size $K$ $=1$ as suggested in Ref. [24] and further explained in Appendix C. This latter approximation is the worst one. This is not surprising as such approximation provides the same result for both the discrete BDE and the continuum ZFE.

For disilicate glass at a lower temperature of $703 \mathrm{~K}$, the critical size is smaller and our approximations deviate more from the numerical solution of the BDE, as shown in Fig. 4(a). Integrating $j(T)$ over time, we find the number of supercritical clusters as a function of time, $N_{c}(t)$, which is depicted in Figs. 3(b) and 4(b). At $703 \mathrm{~K}$, the numerical solution of the BDE with the TF diffusivity yields a time lag $\theta$ $=2.6$. This value is close to those provided by the linearization approximation, $\theta=2.2$, and by Eq. (58), $\theta=2.3$. Thus these analytical approximations to the numerical solution are reasonably good even for a relatively small critical size. However, $\theta=2.6$ gives $1.6 \mathrm{~h}$ according to Table I, whereas the experimentally measured time lag is about $50 \mathrm{~h}$; cf. Fig. 5 of Ref. [3]. This discrepancy is due to having used the TF discrete diffusivity, which yields an excessively small time unit, as shown in Table I.

\section{TEMPERATURE DRIVEN GROWTH OF THE NUCLEUS}

To improve agreement with experiments, we need a discrete diffusivity different from the TF one. We shall no longer assume that cluster size changes due to the activated transfer of a monomer through the cluster surface as in the TF theory. Instead, we shall assume that the discrete diffusivity in the BDE agrees with an adiabatic temperature driven growth of the nucleus. This yields a different formula for $d_{k}$ which, presumably, is not physically justified for very small cluster sizes. Nevertheless, the numerical solution of the BDE corresponding to thermally driven growth provides a time lag which is much closer to the experimentally measured value for disilicate glass than the TF diffusivity.

\section{A. Discrete diffusivity}

Let us assume that there is a nonuniform temperature field about a spherical crystal of radius $a$ ( $k$ cluster) that is growing at the expense of the surrounding glass. Equation (21) shows that a nucleus of critical size grows if $\left(T_{m}-T\right)$ decreases. The same equation yields $\left(T_{m}-T\right)$ at the surface of a critical nucleus with $k$ monomers:

$$
T_{m}-T_{*}=\left(\frac{32 \pi v^{2}}{3}\right)^{1 / 3} \frac{\gamma_{s} N_{A}}{\Delta S_{f}} k^{-1 / 3}
$$

At the surface of the crystal, $T=T_{*}(k)$, whereas far from it there is a smaller temperature, $T=T_{\infty}$. Heat transfer from crystal to glass, $-\rho_{m} c \kappa 4 \pi a^{2} \partial T(a, t) / \partial r\left(\kappa, c\right.$ and $\rho_{m}$ are thermal diffusivity, specific heat and mass density, respectively), should equal the increase of energy due to crystal growth, $T_{\infty} \Delta S_{f} N_{A}^{-1} d k / d t^{*}$. We find 


$$
\frac{d k}{d t^{*}}=-\left.\frac{\rho_{m} c \kappa N_{A}}{T_{\infty} \Delta S_{f}} \frac{\partial T}{\partial r}\right|_{r=a} 4 \pi a^{2} .
$$

The temperature $T(r)$ is the solution of Laplace's equation with boundary conditions $T=T_{*}$ at $r=a$, and $T=T_{\infty}$ infinitely far from the nucleus. The corresponding solution is $T=T_{\infty}$ $+\left(T_{*}-T_{\infty}\right) a / r$, which, together with Eq. (70), yield

$$
\frac{d k}{d t^{*}}=4 \pi \frac{\rho_{m} c \kappa N_{A}}{T_{\infty} \Delta S_{f}}\left(T_{*}-T_{\infty}\right) a .
$$

Using Eq. (69) and the expressions for the radius $a$ and Eq. (21) for the critical size $k_{c}$, we obtain

$$
\frac{d k}{d t^{*}}=\frac{2\left(6 \pi^{2} v\right)^{1 / 3} \rho_{m} c \kappa N_{A}\left(T_{m}-T_{\infty}\right)}{T_{\infty} \Delta S_{f}}\left(k^{1 / 3}-k_{c}^{1 / 3}\right) .
$$

As $k \rightarrow \infty$, the flux (13) in the BDE becomes $j_{k}^{*} \sim d_{k}^{*}\left(e^{-\partial g_{k} / \partial k}\right.$ -1) $\rho_{k}$ (written in dimensional units), and therefore

$$
\frac{d k}{d t^{*}} \sim d_{k}^{*}\left(e^{\varphi-\sigma k^{-1 / 3}}-1\right)=d_{k}^{*} e^{-\sigma k^{-1 / 3}}\left(e^{\varphi}-e^{\sigma k^{-1 / 3}}\right),
$$

Using Eq. (11), this equation can be written as

$$
\frac{d k}{d t^{*}} \sim d_{k}^{*}\left(e^{\varphi\left[1-\left(k_{c} / k\right)^{1 / 3}\right]}-1\right) .
$$

Comparing Eqs. (72) and (73), we obtain

$$
d_{k}^{*}=\Omega k^{1 / 3} \frac{\varphi\left[1-\left(\frac{k_{c}}{k}\right)^{1 / 3}\right]}{e^{\varphi\left[1-\left(k_{c} / k\right)^{1 / 3}\right]}-1}, \quad \Omega=\frac{2\left(6 \pi^{2} v\right)^{1 / 3} \rho_{m} c \kappa k_{B} N_{A}^{2}}{\left(\Delta S_{f}\right)^{2}} .
$$

As before, we shall absorb the constant $\Omega$ in the definition of time according to Eq. (4), which yields the following value of the dimensionless discrete diffusivity:

$$
d_{k}=k^{1 / 3} \frac{\varphi\left[\left(\frac{k_{c}}{k}\right)^{1 / 3}-1\right]}{1-e^{\varphi\left[1-\left(k_{c} / k\right)^{1 / 3}\right]}} .
$$

Here we shall assume that the thermal diffusivity follows the same Arrhenius law as the diffusion coefficient in the liquid $\kappa=D_{0} e^{Q /\left(N_{A} k_{B} T\right)}$; see Kelton et al. [2]. With this choice of discrete diffusivity, a numerical solution of the BDE yields a time lag of $\theta=46.5 \mathrm{~h}$ at $703 \mathrm{~K}$ compared to $\theta=1.6 \mathrm{~h}$ previously obtained using the TF diffusivity. The experimentally measured time lag is $50 \mathrm{~h}$, as shown in Fig. 5, p. 94 of Kelton's review [3]. Thus we feel justified in using our formula (74) to solve the BDE for disilicate glass.

\section{B. Asymptotic theory}

We have to repeat the arguments given in Sec. III using the discrete diffusivity (74) instead of the TF expression. One important difference is that time needs to be rescaled as $T=\epsilon^{2} t$ instead of $T=\epsilon t$. Here we shall also use the symbol $T$ for the slow time scale, but remembering that $T_{T D G}=\epsilon^{2} t$ (for thermally driven growth) instead of $T_{T F}=\epsilon t$ (TF diffusivity).
When necessary, we shall add the labels TF or TDG to the corresponding variables. After the initial discrete stage, our asymptotic theory yields the following results for temperature driven cluster growth, applicable to devitrification of disilicate glass.

The wave front profile $S(X, T)$, with $X=\left[k-\epsilon^{-3} K(T)\right] \epsilon^{3 / 2}$ and $T=\epsilon^{2} t$, is given by $S(X, T)=(1 / 2) \operatorname{erfc}(X /[2 \sqrt{A(T)}])$. The front location and its width solve

$$
\begin{gathered}
\frac{d K}{d T}=U(K) \equiv \widetilde{\varphi}\left(1-K^{1 / 3}\right), \\
\frac{d A}{d T}-2 U^{\prime}(K) A=D(K) \equiv \frac{\widetilde{\varphi}}{2}\left(1-K^{1 / 3}\right) \operatorname{coth}\left[\frac{\widetilde{\varphi}}{2}\left(K^{-1 / 3}-1\right)\right],
\end{gathered}
$$

with initial conditions $K(0)=\epsilon^{3}, A(0)=\epsilon^{3} / 2$. The latter condition corresponds to $q=0$ in Eq. (57). Then $A \sim K / 2$ as $K$ $\rightarrow 0+$, which yields the initial condition for $A$ if $K(0)=\epsilon^{3}$. In Eqs. (76) and (77), $U(K)$ and $D(K)$ are defined by

$$
U(K)=\lim _{\epsilon \rightarrow 0}\left[\epsilon u\left(\frac{K}{\epsilon^{3}}\right)\right], \quad D(K)=\lim _{\epsilon \rightarrow 0}\left[d\left(\frac{K}{\epsilon^{3}}\right)-\frac{1}{2} u\left(\frac{K}{\epsilon^{3}}\right)\right] \epsilon .
$$

Instead of Eq. (49), we get the following approximation for the flux near the wave front:

$$
\begin{aligned}
j_{k} \sim & \frac{\epsilon^{1 / 2} \widetilde{\varphi}\left(1-K^{1 / 3}\right) e^{3 \widetilde{\varphi} /(2 \epsilon)}}{\left[1-e^{-G^{\prime}(K)}\right] \sqrt{4 \pi A}} \exp \left\{-\frac{G(K)}{\epsilon^{3}}-\frac{G^{\prime}(K) X}{\epsilon^{3 / 2}}-G^{\prime}(K)\right. \\
& \left.-\left[\frac{G^{\prime \prime}(K)}{2}+\frac{1}{4 A}\right] X^{2}\right\},
\end{aligned}
$$

in which $G(K)=\widetilde{\varphi}\left(3 K^{2 / 3} / 2-K\right)$ and Eq. (53) has been used. Inserting $X=[1-K(T)] / \epsilon^{3 / 2}$ in this equation, we obtain the nucleation rate:

$$
\begin{aligned}
& \frac{j(T)}{j_{\infty}} \sim \sqrt{\frac{3}{2 A \widetilde{\varphi}}} \frac{U(K)}{1-e^{-G^{\prime}(K)}} \\
& \times \exp \left\{\frac{\widetilde{\varphi}}{2 \epsilon^{3}}-\frac{G(K)+G^{\prime}(K)\left(1+\epsilon^{3}-K\right)}{\epsilon^{3}}\right. \\
&\left.-\left[\frac{G^{\prime \prime}(K)}{2}+\frac{1}{4 A}\right] \frac{(1-K)^{2}}{\epsilon^{3}}\right\}, \\
& j_{\infty}=\sqrt{\frac{\epsilon \widetilde{\varphi}}{6 \pi}} \exp \left(\frac{3 \widetilde{\varphi}}{2 \epsilon}-\frac{\widetilde{\varphi}}{2 \epsilon^{3}}\right) .
\end{aligned}
$$

The simplest approximation for the nucleation rate and the time lag yields

$$
\begin{gathered}
j=j_{\infty} \exp \left\{-e^{-\left(t-t_{M}\right) / \tau}\right\}, \\
t_{M}=\frac{1}{\widetilde{\varphi} \epsilon^{2}} \int_{\epsilon^{3}}^{1-\epsilon^{3 / 2} \kappa_{M}} \frac{d K}{1-K^{1 / 3}} \\
=\frac{3}{2 \widetilde{\varphi} \epsilon^{2}}\left\{\ln \left(\frac{3 \widetilde{\varphi}}{2 \epsilon^{3}}\right)-3+2\left(\frac{6 \epsilon^{3}}{\widetilde{\varphi}}\right)^{1 / 2}\right\}+O(\epsilon),
\end{gathered}
$$



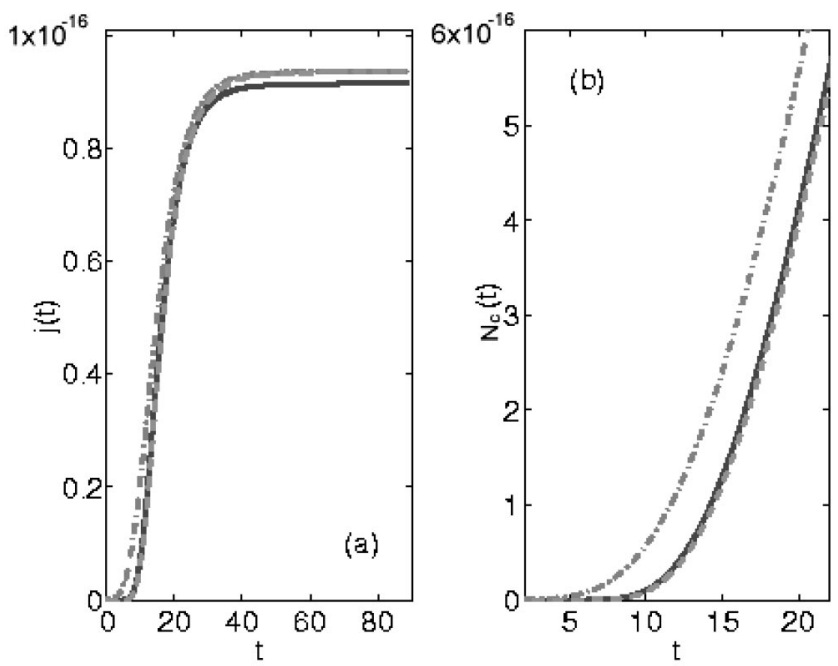

FIG. 5. (a) Evolution of the dimensionless flux at critical size $j(t)$, and (b) number of clusters surpassing critical size $N_{c}(t)$ as a function of time (in dimensionless units) for disilicate glass at $820 \mathrm{~K}, k_{c}=34$. Solid lines correspond to numerical results, dashed lines to the approximation given by Eqs. (76)-(79), and dot-dashed lines to Eqs. (81)-(83).

$$
\begin{gathered}
\tau_{T D G}^{-1}=\frac{2 \widetilde{\varphi} \epsilon^{2}}{3}=\epsilon \tau_{T F}^{-1}, \\
\theta=t_{M}+\left[\gamma+E_{1}\left(e^{t_{M} / \tau}\right)\right] \tau \\
=\frac{3}{2 \tilde{\varphi} \epsilon^{2}}\left\{\ln \left(\frac{3 \tilde{\varphi}}{2 \epsilon^{3}}\right)-3+\gamma+2\left(\frac{6 \epsilon^{3}}{\tilde{\varphi}}\right)^{1 / 2}\right\}+O(\epsilon) .
\end{gathered}
$$

To obtain these expressions, we have followed the same procedure as in the case of the TF diffusivity. In particular, Eqs. (61)-(63) hold with $T=\epsilon^{2} t, t_{M}$ given by Eq. (82) and $\tau$ given by Eq. (83).

Figure 5 compares the numerical solution of the BDE (solid line) for devitrification of disilicate glass at $820 \mathrm{~K}$ (critical size $k_{c}=34$ ) with the more accurate asymptotic formulas: Eqs. (81)-(83) (dot-dashed line) and its linearization about the critical size, Eqs. (76)-(79) (dashed line). Similarly, Fig. 6 corresponds to $703 \mathrm{~K}$. We observe that our two approximations, Eqs. (76)-(79), and Eq. (81), describe quite accurately the numerical solution. Notice that our asymptotic formulas for thermally driven growth yield worse approximations to the numerical solution of the BDE than in the case of the TF diffusivity. The stationary nucleation rate is approximated less well by $j_{\infty}$ in the case of thermally driven growth because of the avoidable singularity of $d_{k}$ at the integer $k_{c}$, which is slightly different from $\epsilon^{-3}$.

\section{DISCUSSION}

In this paper, we have studied the case of phase segregation resulting when $\rho>\rho_{c}$. Previously, other authors had carried out asymptotic studies of the BDE in the simpler case of subcritical density, $\rho<\rho_{c}$, in which initial conditions of only
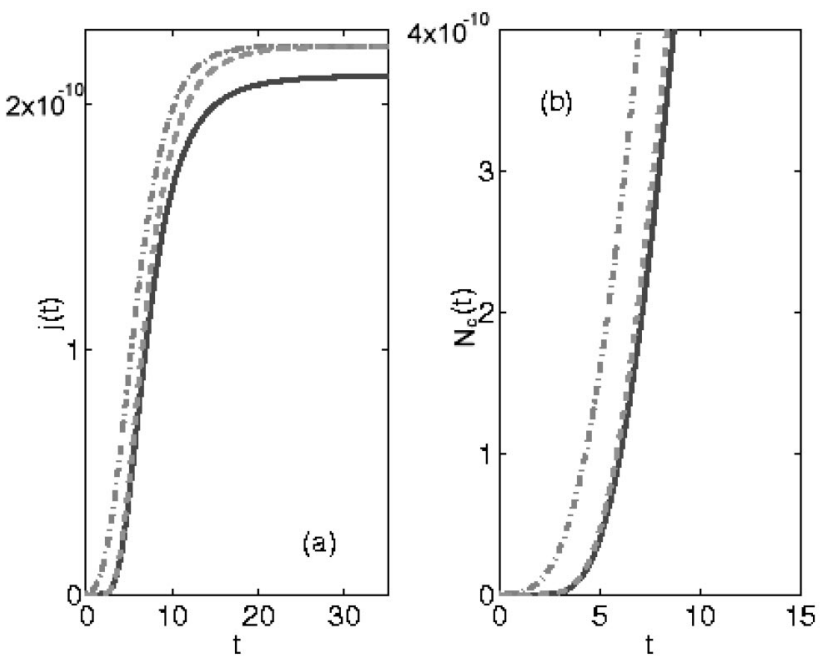

FIG. 6. Same as Fig. 5 for disilicate glass at $703 \mathrm{~K}$, which has a critical size $k_{c}=18$.

monomers, or more general ones, evolve towards the equilibrium distribution. In many cases of polynomial growth for $d_{k}$, equilibrium is reached via a wave front profile for $s_{k}$, which is similar to Eq. (54) with $A \propto K^{\delta}$, and $K \propto T^{\mu}$, for appropriate positive $\delta$ and $\mu$; see Ref. [25] and references cited therein. This advancing and widening wave front leaves in its wake the equilibrium size distribution.

In the more complex case of phase segregation and indefinite aggregate growth considered in this paper, a quasicontinuum wave front of $s_{k}$ emerges after a short transient which is governed by the discrete BDE. After this, the leading edge of the wave front advances towards the critical size, and it slows down and stops there, leaving behind it a quasiequilibrium state. The arrival of the wave front to the critical size marks the ignition of nucleation of supercritical clusters, which ends when the stationary Zeldovich rate is reached. Previous asymptotic theories have been derived for the continuum ZFE, not the discrete BDE, and thus their results systematically misrepresent two things: (i) the time lags for transient nucleation, as explained by $\mathrm{Wu}$ [19], and (ii) the width of the wave front and the time to ignition in the nucleation rate. The latter discrepancies occur because the diffusion coefficient appearing in the continuum equation for the wave front satisfies $D_{B D E}(K)=D_{Z F E}(K)-U(K) / 2$, and therefore the width of the ignition stage (or of the wave front) for the BDE is smaller than the corresponding one for the ZFE.

Let us briefly mention several existing asymptotic theories for the ZFE. Shneidman [21] and Shi et al. [22] Laplace transformed the continuum ZFE and matched a first stage of pure advection of clusters to a local expansion about the wave front when it is near its final position at the critical size. They obtained our simplest formula for the nucleation rate, Eq. (67) with the same relaxation time, $\tau_{T F}$ or $\tau_{T D G}$, except that their values for $t_{M}$ were different from Eq. (66). This can be expected from Wu's arguments about approximating the discrete BDE by the continuum ZFE [19]; see the systematic shift of approximations of the ZFE with respect to numerical solutions of the BDE in Fig. 20 of Ref. [19]. Trinkaus and Yoo [20] studied a ZFE with a drift term lin- 
earized about the critical size (parabolic barrier) as an approximation to the full ZFE. Their results are comparable to those found by means of the Laplace transform and matched asymptotic expansions; see Wu's review [19]. All these authors obtained a transition region for the nucleation rate $j(t)$ that was wider than observed in the numerical solution of the BDE. Several authors also found a nucleation rate for supercritical clusters that did not tend to $j_{\infty}$ as $t \rightarrow \infty$ if $k \neq k_{c}$ $[20,22,24]$, which is often called the asymptotics catastrophe [26]. Our theory is free from this deficiency: Eq. (B5) in Appendix B provides the flux at $k>k_{c}$ using the TF diffusivity

$$
\begin{aligned}
j_{k} & =j_{\infty} e^{-\widetilde{\varphi} X_{0} \kappa / 3} e^{-\widetilde{\varphi} \kappa^{2} / 6} \\
& =j_{\infty} \exp \left[-X_{0} \sqrt{2 \widetilde{\varphi} / 3} e^{-\left(t-t_{M}\right) /(2 \tau)}\right] e^{-e^{-\left(t-t_{M}\right) / \tau}},
\end{aligned}
$$

in which $X_{0}=\epsilon^{3 / 2}\left(k-k_{c}\right)$. Notice that $j_{k} \sim j_{\infty}$ as $t \rightarrow \infty$, even after making our simplest approximation: linearization of the wave front about the critical size. To get rid of the asymptotics catastrophe, Maksimov et al. [26] assumed that $S(X, T)=(1 / 2) \operatorname{erfc}\left\{\left[A e^{-t /(2 \tau)}+B(X)\right] / \sqrt{1-\zeta e^{-t / \tau}}\right\}$, in which the new function $B(X)$ obeyed an ad hoc self-consistent equation that ensured $j_{k} \sim j_{\infty}$ as $t \rightarrow \infty$ even if $k \neq k_{c}$. Note that if we use Eq. (63) for $X=\kappa$ and the linearization approximation for $A$ as in Appendix C, we obtain the previous formula for $S$ with $\zeta=1, A=e^{t_{M} /(2 \tau)}$ and $B=0$. Shneidman [27] criticized Maksimov et al.'s result and extended his earlier asymptotic formula for the nucleation rate [23] to noncritical sizes. The previous criticism of using approximations to the ZFE instead of approximations to the discrete BDE apply to these works. Our more precise approximation using Eq. (58) plus the exact equations for the wave front location and its instantaneous width improve upon these approximations and perform better for materials with large critical sizes.

The time lag obtained from the numerical solution of the BDE with the TF diffusivity (or from our asymptotic approximations using it) is too small as compared with experimental results (about thirty times smaller for disilicate at $703 \mathrm{~K})$. The TF discrete diffusivity yields an excessively small time unit, as shown in Table I. We have greatly improved the agreement of theory and experiments by using a different formula for the discrete diffusivity, which is found by imposing that the growth rate of a critical nucleus resulting from the BDE be the same as obtained by heat transfer. In this case, our asymptotic approximations have a slightly different scaling of time and different expressions for $U(K)$ and $D(K)$.

\section{ACKNOWLEDGMENTS}

Part of this work was carried out during a visit of J.N.'s to the Universidad Carlos III de Madrid, whose support we acknowledge. The present work was financed by the Spanish MCyT Grants No. BFM2002-04127-C02-01 and No. BFM2002-04127-C02-02, and by the European Union under Grant No. HPRN-CT-2002-00282.

\section{APPENDIX A: GENERAL SOLUTION OF EQ. (51)}

It is convenient to rewrite this equation in terms of the variables $K$ and $X$, as

$$
\frac{\partial J}{\partial K}+\frac{U^{\prime}(K)}{U(K)} \frac{\partial(X J)}{\partial X}=\frac{D(K)}{U(K)} \frac{\partial^{2} J}{\partial X^{2}},
$$

to be solved with the homogeneous boundary condition

$$
\left(\epsilon^{-3 / 2}+\frac{U^{\prime}(K) X_{i n}}{U(K)}\right) J-\frac{D(K)}{U(K)} \frac{\partial J}{\partial X}=0
$$

(at $X=X_{\text {in }} \equiv \epsilon^{3 / 2}-K \epsilon^{-3 / 2}$, corresponding to $k=1$ in the definition of $X)$, and with initial condition $J\left(X, K_{0}\right)$ $=-\partial S_{0}(X) / \partial X$. The boundary condition is obtained by differentiating

$$
S\left(X_{i n}, T\right)=1
$$

with respect to $T$ and then using the definition of $J$ and Eqs. (41) and (47). The solution of the initial-boundary value problem is

$$
J(X, K)=-\int_{-\infty}^{\infty} G\left(X, K ; X_{0}, K_{0}\right) \frac{\partial S_{0}\left(X_{0}\right)}{\partial X_{0}} d X_{0},
$$

where the Green's function $G\left(X, K ; X_{0}, K_{0}\right)$ satisfies Eq. (A1) with initial condition $G\left(X, K_{0}+; X_{0}, K_{0}\right)=\delta\left(X-X_{0}\right)$ and the same homogeneous boundary condition as $J$ at $X=X_{i n}$. In a simple application of the method of images, the Green's function for this BVP can be written in terms of the Green's function $G_{\infty}\left(X, K ; X_{0}, K_{0}\right)$ for the infinite real line $X$, as

$$
\begin{aligned}
G\left(X, K ; X_{0}, K_{0}\right)= & G_{\infty}\left(X, K ; X_{0}, K_{0}\right) \\
& +c\left(X_{0} ; K, K_{0}\right) G_{\infty}\left(X, K ; 2 X_{i n} U_{0} / U-X_{0}, K_{0}\right),
\end{aligned}
$$

$$
c\left(X_{0} ; K, K_{0}\right)=\frac{\frac{\left(\frac{X_{\text {in }}}{U}-\frac{X_{0}}{U_{0}}\right) D}{\left(\epsilon^{-3 / 2}+\frac{U^{\prime} X_{i n}}{U}\right) 2 U B}+1}{\frac{\left(\frac{X_{\text {in }}}{U}-\frac{X_{0}}{U_{0}}\right) D}{\left(\epsilon^{-3 / 2}+\frac{U^{\prime} X_{\text {in }}}{U}\right) 2 U B}-1}
$$

$$
B\left(K, K_{0}\right)=\int_{K_{0}}^{K} \frac{D(K) d K}{U(K)^{3}} .
$$

Now, $G_{\infty}\left(X, K ; X_{0}, K_{0}\right)$ can be calculated by first writing an equation for the Fourier transform $\hat{G}_{\infty}\left(\xi, K ; X_{0}, K_{0}\right)$ $=\int_{-\infty}^{\infty} e^{i \xi X} G_{\infty}\left(X, K ; X_{0}, K_{0}\right) d X$. Such an equation is a first-order quasilinear hyperbolic equation that can be solved by the method of characteristics. The result is that $\hat{G}_{\infty}$ is Gaussian in $\xi$. Inverting the Fourier transform, we obtain 


$$
G_{\infty}\left(X, K ; X_{0}, K_{0}\right)=\frac{e^{-\left(X / U-X_{0} / U_{0}\right)^{2} / 4 B\left(K, K_{0}\right)}}{U \sqrt{4 \pi B\left(K, K_{0}\right)}} .
$$

Given the initial condition $S_{0}\left(X_{0}\right)=H\left(2 \epsilon^{3 / 2}-K_{0} \epsilon^{-3 / 2}-X_{0}\right)$ (pure monomers), Eq. (A4) yields

$$
J(X, K)=G\left(X, K ; 2 \epsilon^{3 / 2}-\epsilon^{-3 / 2} K_{0}, K_{0}\right) \sim \frac{e^{-X^{2} / 4 U^{2} B\left(K, K_{0}\right)}}{U \sqrt{4 \pi B\left(K, K_{0}\right)}},
$$

which is Eq. (53), up to exponentially small terms. Here $K_{0}=\epsilon^{3}, U_{0}=U\left(K_{0}\right)=2 \epsilon^{2} \sinh [\widetilde{\varphi} /(2 \epsilon)-\widetilde{\varphi} / 2] \sim \epsilon^{2} e^{\widetilde{\varphi} /(2 \epsilon)-\tilde{\varphi} / 2}, U$ $=U(K)$, and $K=K(T)$.

\section{APPENDIX B: CALCULATING THE TIME LAG}

The time $T_{M}$ can be estimated from Eq. (41) with initial condition $K(0)=\epsilon^{3}$ (pure monomers) as

$$
\begin{aligned}
T_{M}-\epsilon t_{\infty}= & \int_{\epsilon^{3}}^{1-\epsilon^{3 / 2} \kappa_{M}} \frac{d K}{U(K)} \\
= & \int_{\epsilon^{3}}^{1-\epsilon^{3 / 2} \kappa_{M}} \frac{d K}{U^{\prime}(1)(K-1)} \\
& +\int_{\epsilon^{3}}^{1-\epsilon^{3 / 2} \kappa_{M}}\left[\frac{1}{U(K)}-\frac{1}{U^{\prime}(1)(K-1)}\right] d K,
\end{aligned}
$$

where $t_{\infty}$ is the duration of the initial discrete stage in the original time scale. After straightforward calculations, we obtain Eq. (66).

The number of supercritical clusters is

$$
\begin{aligned}
N_{c} \sim \int_{0}^{t} j(t) d t & =j_{\infty}\left\{t+\int_{0}^{t}\left[\exp \left(-e^{-\left(t-t_{M}\right) / \tau}\right)-1\right] d t\right\} \\
& \sim j_{\infty}(t-\theta), \\
\theta & \equiv \int_{0}^{\infty}\left[1-\exp \left(-e^{-\left(t-t_{M}\right) / \tau}\right)\right] d t \\
& =\tau \int_{0}^{e^{t_{M} / \tau}} \frac{1-e^{-x}}{x} d x=t_{M}+\tau \gamma+\tau E_{1}\left(e^{t_{M} / \tau}\right),
\end{aligned}
$$

where $E_{1}(x)$ is an exponential integral and $\gamma=0.577215 \ldots$ is Euler's constant [28]. Notice that $\tau E_{1}(x) \sim \tau e^{-x} / x \sim 9 \epsilon[\widetilde{\varphi}(1$ $\left.\left.-\epsilon^{3}\right)\right]^{-2} e^{-\widetilde{\varphi} /\left(6 \epsilon^{3}\right)} \ll \epsilon \ll 1$, as $x=e^{t_{M} / \tau} \sim \widetilde{\varphi} /\left(6 \epsilon^{3}\right) \gg 1$ [28]. Thus we can ignore the exponential integral in Eq. (B3), which simplifies somewhat more Eq. (68).

Sometimes it is interesting to calculate the creation rate of clusters of size $k>k_{c}$. If $k$ is close to critical size, we can write

$$
X=\epsilon^{3 / 2}\left(k-\frac{K}{\epsilon^{3}}\right)=\epsilon^{3 / 2}\left(k-\epsilon^{-3}\right)+\kappa .
$$

Thus $X=X_{0}+\kappa$, with $X_{0}=\epsilon^{3 / 2}\left(k-\epsilon^{-3}\right)$. Inserting $X=X_{0}+\kappa$ and $K=1-\epsilon^{3 / 2} \kappa$ in Eq. (58), we obtain the creation rate of clusters of size $k=\epsilon^{-3}+X_{0} \epsilon^{-3 / 2}$ :

$$
\begin{aligned}
j_{k} & =j_{\infty} e^{-\widetilde{\varphi} X_{0} \kappa / 3} e^{-\widetilde{\varphi} \kappa^{2} / 6} \\
& =j_{\infty} \exp \left[-X_{0} \sqrt{2 \widetilde{\varphi} / 3} e^{-\left(t-t_{M}\right) /(2 \tau)}\right] e^{-e^{-\left(t-t_{M}\right) / \tau}} .
\end{aligned}
$$

Notice that $j_{k} \sim j_{\infty}$ as $t \rightarrow \infty$. Thus our asymptotic result for the flux over any cluster size is free from the asymptotics catastrophe [26]: several authors found that their expressions for the flux tend to $J \neq j_{\infty}$ as $t \rightarrow \infty$ if $k \neq k_{c}$. These catastrophes are due to inappropriate assumptions they made in their derivations.

\section{APPENDIX C: LINEARIZATION OF THE EQUATIONS FOR $K(T)$ AND $A(T)$ ABOUT THE CRITICAL SIZE}

A possible approximation of the wave front equations consists of linearizing the equations for $K(T)$ and $A(T)$ about the critical size $K=1$ :

$$
\begin{gathered}
\frac{d K}{d T} \approx U^{\prime}(1)(K-1), \\
\frac{d A}{d T}-2 U^{\prime}(1) A \approx D(1),
\end{gathered}
$$

with $K(0)=\epsilon^{3}$ and $A(0) \approx 0$ (initial condition of pure monomers). Both for the TF discrete diffusivity and for temperature driven growth of the nucleus, we have

$$
U^{\prime}(1)=-\frac{\widetilde{\varphi}}{3}, \quad D(1)=1 .
$$

The solutions of Eqs. (C1) and (C2) can be written in terms of the time scale $t$ as

$$
\begin{gathered}
K(t) \approx 1-\left(1-\epsilon^{3}\right) e^{-t /(2 \tau)}, \\
A(t) \approx \frac{3}{2 \widetilde{\varphi}}\left(1-e^{-t / \tau}\right),
\end{gathered}
$$

for the TF diffusivity, and the same formulas with $\tau_{T D G}$ instead of $\tau_{T F}$ for thermally driven growth. Near the critical size, these equations would give an explicit expression of the wave front profile (54), with $X=\epsilon^{3 / 2} k-\epsilon^{-3 / 2} K(T)$. The nucleation rate of supercritical clusters is then obtained from Eq. (58) with $X=(1-K) / \epsilon^{3 / 2}$, together with Eqs. (C4) and (C5). For the TF diffusivity, $j \equiv j_{k_{c}}$ is

$$
\begin{aligned}
\frac{j}{j_{\infty}} \sim & \frac{\left[1-\left(1-\epsilon^{3}\right) e^{-t /(2 \tau)}\right]^{2 / 3}}{\sqrt{1-e^{-t / \tau}}} \\
& \times \exp \left\{-\widetilde{\varphi}\left[\frac{\Psi(t)}{\epsilon^{3}}+\frac{\left(1-\left(1-\epsilon^{3}\right) e^{-t /(2 \tau)}\right)^{-1 / 3}-1}{2}\right]\right\},
\end{aligned}
$$

$$
\begin{aligned}
\Psi(t)= & {\left[1-\left(1-\epsilon^{3}\right) e^{-t /(2 \tau)}\right]^{2 / 3}+\left[1-\left(1-\epsilon^{3}\right) e^{-t /(2 \tau)}\right]^{-1 / 3}-2 } \\
& +\frac{\left(1-\epsilon^{3}\right)^{2} e^{-t / \tau}}{6}\left\{\frac{1}{1-e^{-t / \tau}}-\left[1-\left(1-\epsilon^{3}\right) e^{-t /(2 \tau)}\right]^{-4 / 3}\right\} .
\end{aligned}
$$


Notice that the same results would have been obtained from the ZFE because the difference between our $D(K)$ and the corresponding one for the ZFE is $U(K) / 2$, which vanishes at $K=1$. If we replace 1 instead of $\left[1-\left(1-\epsilon^{3}\right) e^{-t /(2 \tau)}\right]$ in the previous formulas, we find

$$
j \sim \frac{j_{\infty}}{\sqrt{1-e^{-t / \tau}}} \exp \left\{-\frac{\tilde{\varphi}\left(1-\epsilon^{3}\right)^{2}}{6 \epsilon^{3}} \frac{e^{-2 t / \tau}}{1-e^{-t / \tau}}\right\} .
$$

Demo and Kozísek's theory for the ZFE [24] would yield Eq. (C8) for the nucleation rate once a couple of errors are corrected. They found $\tau_{D K}=7 \tau_{T F} / 5$ instead of the correct relax- ation time $\tau_{T F}$, and an extra factor of 9 in the argument of the exponential in Eq. (C8). Moreover, their exponential contains a factor $(1-\epsilon)$ instead of $\left(1-\epsilon^{3}\right)$. Demo and Kozísek's Fig. 3 shows that their formulas do not improve as the cluster size increases, as one would expect of correct asymptotic expressions. Instead, they seem to optimize the nucleation rate of crystals in disilicate at $k_{c}=27(T=800 \mathrm{~K})$, as compared with numerical solutions. The earlier theory by Trinkaus and Yoo [20] calculated the Green function for a time-dependent ZFE with a quadratic barrier and also used a linear equation for the position of the wave front. Thus their results are related to those in this appendix.
[1] E. M. Lifshitz and L. P. Pitaevskii, Physical Kinetics (Pergamon, New York, 1981).

[2] K. F. Kelton, A. L. Greer, and C. V. Thompson, J. Chem. Phys. 79, 6261 (1983).

[3] K. F. Kelton, in Solid State Physics, edited by H. Ehrenreich and D. Turnbull (Academic, New York, 1991), Vol. 45, p. 75.

[4] Mathematical Modelling for Polymer Processing, Mathematics in Industry Vol. 2, edited by V. Capasso (Springer, Berlin, 2003).

[5] U. Gasser, E. R. Weeks, A. Schofield, P. N. Pursey, and D. A. Weitz, Science 292, 258 (2001).

[6] J. N. Israelachvili, Intermolecular and Surface Forces, 2nd ed. (Academic, New York, 1991).

[7] J. C. Neu, J. A. Cañizo, and L. L. Bonilla, Phys. Rev. E 66, 061406 (2002).

[8] I. M. Lifshitz and V. V. Slyozov, J. Phys. Chem. Solids 19, 35 (1961).

[9] S. Q. Xiao and P. Haasen, Acta Metall. Mater. 39, 651 (1991).

[10] S. P. Marsh and M. E. Glicksman, Acta Mater. 44, 3761 (1996).

[11] O. Penrose, J. Stat. Phys. 89, 305 (1997).

[12] J. J. L. Velázquez, J. Stat. Phys. 92, 195 (1998).

[13] B. Niethammer, J. Nonlinear Sci. 13, 115 (2003).

[14] O. Penrose and A. Buhagiar, J. Stat. Phys. 30, 219 (1983); O.
Penrose, J. L. Lebowitz, J. Marro, M. Kalos, and J. Tobochnik, ibid. 34, 399 (1984).

[15] V. Ganesan and H. Brenner, Phys. Rev. E 59, 2126 (1999).

[16] D. Turnbull and J. C. Fisher, J. Chem. Phys. 17, 71 (1949).

[17] Ya. B. Zeldovich, Zh. Eksp. Teor. Fiz. 12, 525 (1942); Acta Physicochim. URSS 18, 1 (1943).

[18] D. Kashchiev, Surf. Sci. 14, 209 (1969); 18, 389 (1969).

[19] D. T. Wu, Solid State Phys. 50, 37 (1996).

[20] H. Trinkaus and M. H. Yoo, Philos. Mag. A 55, 269 (1987).

[21] V. A. Shneidman, Zh. Tekh. Fiz. 57, 131 (1987) [Sov. Phys. Tech. Phys. 32, 76 (1987)]; 58, 2202 (1988) [33, 1338 (1988)].

[22] G. Shi, J. H. Seinfeld, and K. Okuyama, Phys. Rev. A 41, 2101 (1990); 44, 8443 (1991). Higher order approximation in J. J. Hoyt and G. Sundar, Scr. Metall. Mater. 29, 1535 (1993).

[23] V. A. Shneidman, Phys. Rev. A 44, 2609 (1991).

[24] P. Demo and Z. Kozísek, Phys. Rev. B 48, 3620 (1993).

[25] J. R. King and J. A. D. Wattis, J. Phys. A 35, 1357 (2002).

[26] I. L. Maksimov, M. Sanada, and K. Nishioka, J. Chem. Phys. 113, 3323 (2000).

[27] V. A. Shneidman, J. Chem. Phys. 115, 8141 (2001).

[28] M. Abramowitz and I. A. Stegun, Handbook of Mathematical Functions (Dover, New York, 1965). 